\title{
Inhaled Corticosteroids, Corticosteroid Osteoporosis, and the Risk of Fracture in Chronic Asthma
}

\author{
John H. Toogood, MD, FRCPC
}

\begin{abstract}
Current guidelines for the diagnosis and treatment of osteoporosis do not address the risks to bone density and the likelihood of fracture that may be associated with inhaled corticosteroid treatment for asthma. This review outlines an approach to the use of bone densitometry in clinical practice for the diagnosis, prevention, and treatment of osteoporosis in asthmatic patients receiving inhaled corticosteroid therapy.
\end{abstract}

\section{Diagnosis of Osteoporosis}

Bone densitometry by dual-energy x-ray absorptiometry (DXA) allows osteoporosis to be diagnosed and corrective treatment initiated before clinical fractures occur. ${ }^{1}$ The hip and lumbosacral spine are the best validated sites for an objective measurement of bone mineral density (BMD) from which to estimate fracture risk. The degree of risk is estimated to increase 1.5- to 3-fold for every standard deviation decrease in BMD.

To facilitate clinical interpretation of the test result, BMD measured in absolute terms is converted to a $\mathrm{T}$-score and/or Z-score. The T-score compares the patient's measured BMD with the average value for healthy young adults when their peak bone mass is normally attained (about

\footnotetext{
J. H. Toogood - Emeritus Professor of Medicine, University of Western Ontario, Division of Clinical Immunology and Allergy, Department of Medicine, London Health Sciences Centre, London, Ontario

Correspondence to: Dr. John H. Toogood, 194 Hunt Club Drive, London, ON N6H 3 Y9
}

30 years of age). T-score values at or below -2.5 indicate osteoporosis and a clinically important increase in fracture risk. ${ }^{2}$ The Z-score compares the patient's measured BMD with that of a population of healthy persons of the same age and sex as the patient, that is, it "controls" for the potentially confounding effects of concurrent reductions in BMD that relate to advancing age.

Decisions about the need for preventive or corrective antiosteoporosis treatment in a particular patient or patient group require that the presence or absence of clinical risk factors for fracture be taken into consideration, as well as the BMD measurement score. ${ }^{3}$ Relevant risk factors include the patient's age ( $>$ or $<65$ years) and current and past exposure to corticosteroid (glucocorticoid) therapy (Table 1).

DXA is recommended for patients who have been exposed to systemic corticosteroid therapy for 3 months or longer. ${ }^{3}$ The current Canadian guidelines for the management of osteoporosis do not cite inhaled corticosteroid (ICS) therapy as a risk factor that merits densitometry (see Table 1).

\section{Contrary Effects of ICS Therapy on Bone Density and the Risk of Fracture}

Low daily doses of ICS administered to adults for $10 \pm 5.5$ years (Figure 1$)^{4}$ or to children for 4 to 6 years $^{5}$ did not significantly reduce BMD. However, high-dose ICS therapy has been shown to reduce $\mathrm{BMD}$ in adults with asthma ${ }^{4}$ or chronic obstructive pulmonary disease ${ }^{6}$ and in premenopausal asthmatic women. ${ }^{7}$

As much as $45 \%$ of the variability in BMD that may be demonstrable among elderly asthmatic patients cannot be explained on the basis of their 
Table 1 Factors That Identify People Who Should Be Assessed for Osteoporosis

\begin{tabular}{ll}
\hline Major Risk Factors & Minor Risk Factors \\
\hline Age $>65 \mathrm{yr}$ & Rheumatoid arthritis \\
Vertebral compression fracture & Past history of clinical hyperthyroidism \\
Fragility fracture after age $40 \mathrm{yr}$ & Chronic anticonvulsant therapy \\
Family history of osteoporotic fracture & Low dietary calcium intake \\
(especially maternal hip fracture) & Smoker \\
Systemic glucocorticoid therapy & Excessive alcohol intake \\
of $>3$ mo duration & Excessive caffeine intake \\
Malabsorption syndrome & Weight $<57$ kg \\
Primary hyperparathyroidism & Weight loss $>10 \%$ of weight at age $25 \mathrm{yr}$ \\
Propensity to fall & Chronic heparin therapy \\
Osteopenia apparent on radiograph & \\
Hypogonadism & \\
Early menopause (before age $45 \mathrm{yr})$ & \\
\hline
\end{tabular}

Adapted from Brown JP et al. ${ }^{3}$

past or current exposures to corticosteroid therapy or other clinically identifiable risk factors for osteoporosis. ${ }^{4,8}$ Genetic determinants, as yet not identified, are thought to account for much of this variability. Some identifiable factors that may act to conserve or restore BMD in asthmatic adults receiving long-term ICS therapy, and thereby reduce their risk of fracture, are shown in Table 2.

A cross-sectional survey by DXA in asthmatic adults previously treated with prednisone found that higher current daily doses of ICS were associated with reduced Z-score values for BMD (see Figure 1), whereas in the same patient group, larger cumulative lifetime exposures to ICS correlated paradoxically with higher BMD values (see Table 2) and a corollary, clinically important, dose-related reduction in the numbers of patients at risk of fracture (Table 3). ${ }^{4}$ This illustrates the fact that despite prolonged and continuing exposure to prednisone, osteoporosis remains a potentially reversible process, and substituting ICS for current prednisone use may, in many patients, conserve or improve BMD sufficiently to reduce the risk of fracture to a level approximating that of healthy persons of the same age and sex who have never been exposed to inhaled or oral corticosteroid therapy (see Table 3). Prednisone withdrawal and increased physical activity consequent to improved asthma control may be proximate causes of this favourable outcome of ICS treatment (see Table 2).

\section{Prevention and Treatment of Glucocorticoid-Induced Osteoporosis}

It is recommended that the presence or absence of clinical risk factors for osteoporosis and fracture

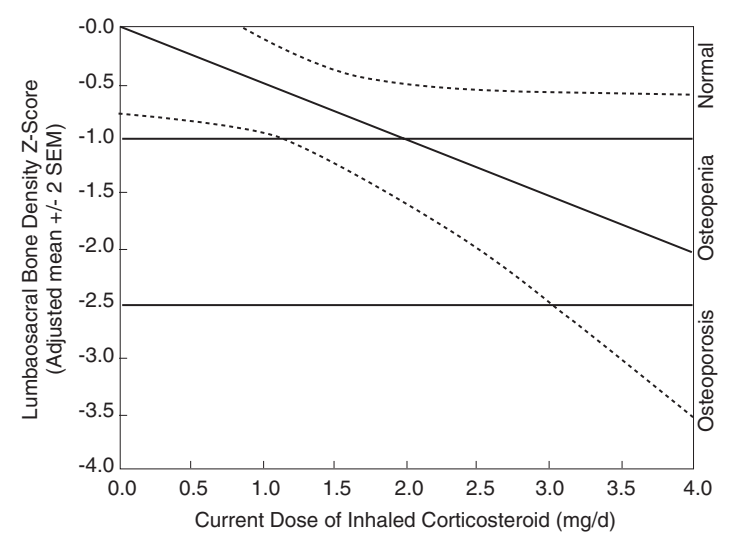

Figure 1 Regression of lumbosacral bone mineral density on the daily dose of inhaled corticosteroid in a crosssectional analysis of bone density in 69 corticosteroiddependent adults with moderate to severe chronic asthma. ${ }^{4}$ The calculated mean regression ( $\pm 95 \%$ confidence limits) is adjusted to control for the effects of age, sex, years of estrogen use, physical activity, the current daily dose of prednisone, years of prednisone use, and the cumulative lifetime dose of inhaled corticosteroid. Bone density declined significantly on the current daily dose of inhaled corticosteroid ( $p=.013$ analysis of covariance). At doses $\leq 1.0 \mathrm{mg} / \mathrm{d}$, bone density did not differ significantly from normal. SEM $=$ standard error the mean. 
Table 2 Correlates of Higher Lumbosacral Bone Density Z-Score Values in ICS-Treated Asthmatic Adults*

\begin{tabular}{lc}
\hline Correlates of Higher Bone Density & $\boldsymbol{p}$ Value (ANCOVA) \\
\hline Larger cumulative lifetime dose of ICS & .002 \\
Lower current daily dose of ICS & .013 \\
Fewer years of prednisone exposure & .032 \\
Greater physical activity & .042 \\
More years of supplemental estrogen use & .058 \\
\hline
\end{tabular}

Adapted from Toogood $\mathrm{JH}$ et al. ${ }^{4}$

ANCOVA $=$ analysis of covariance; ICS $=$ inhaled corticosteroid.

$* N=69$. Age: $59.9 \pm 13.3 \mathrm{yr}(\mathrm{SD})$.

Years of steroid exposure $($ mean $\pm \mathrm{SD})$ : prednisone $=10.7 \pm 9.7 \mathrm{yr}$, ICS $=10.1 \pm 5.5 \mathrm{yr}$.

be documented and a baseline DXA measurement secured in patients who are commencing ICS therapy and appear likely to need a maintenance dose $>1.0 \mathrm{mg}$ beclomethasone dipropionate (BDP) per day or a systemically equivalent dose of an alternative ICS. . Depending on the DXA result and clinical considerations, appropriate antiosteoporosis therapy may be indicated.

Current guidelines recommend that all patients receiving glucocorticoid therapy take an oral calcium supplement daily (at least $1,000 \mathrm{mg}$ of $\mathrm{Ca}^{++}$) plus activated vitamin $\mathrm{D}_{2} \cdot{ }^{3}$ For patients who use medium to high doses of glucocorticoid, activated vitamin $\mathrm{D}_{3}$ (cholecalciferol) is preferred over vitamin $\mathrm{D}_{2}$ because the latter is less potent.

In asthma patients deemed to be potentially at risk despite normal BMD, an orally administered bisphosphonate antiresorptive agent (etidronate, alendronate, risedronate) may be indicated to prevent bone loss.,.$^{2,310,11}$ In patients who are already at risk because of established glucocorticoid-induced osteoporosis, bisphosphonates have the capacity to restore bone mass and thus reduce the risk of vertebral fracture. ${ }^{3}$ They are currently recommended as first-line therapy for the prevention or treatment of osteoporosis in men or postmenopausal women who use prednisone at doses $\geq 5 \mathrm{mg} / \mathrm{d} .{ }^{3}$ However, bisphosphonates are generally not appropriate for young women or children. ${ }^{12}$ Bisphosphonates and calcium should not be taken at the same time of day because the calcium impairs absorption of the bisphosphonate.

\section{Therapeutic Tactics}

It is important that the daily dose of ICS be adjusted individually for each patient to ensure that it is adequate to fully control day-to-day asthma symptoms, to eliminate the need for long-term prednisone, and to prevent exacerbations of asthma that trigger periodic intervention with high and potentially bone-depleting doses of prednisone. The bone-depleting effect of episodic high-dose prednisone use has been shown to persist long after the transient high-dose regimen has been terminated. ${ }^{13}$

The daily dose of ICS should be sufficient to facilitate daily weight-bearing (impact type) physical activity. ${ }^{14} \mathrm{~A}$ regular exercise regimen suited to the particular needs and capacity of each patient should be encouraged. ${ }^{3}$

With a high-potency antiasthmatic ICS such as fluticasone, most patients achieve an optimal therapeutic response with a dose $\leq 0.5 \mathrm{mg} / \mathrm{d} . .^{15,16}$ Higher doses increase the risk of adverse systemic effects disproportionately. ${ }^{15}$

For patients who need a high daily dose of ICS to ensure optimum control of unstable asthma, the current treatment of choice is a combination formulation such as fluticasone plus salmeterol ${ }^{17}$ or budesonide plus formoterol. ${ }^{18}$ Administered twice daily, these products offer the advantage that equivalent asthma control may be achieved with a lower and safer daily dose of ICS. ${ }^{17,18}$

Alternatively, in patients with unstable moderate to severe chronic asthma, administering 
Table 3 Cumulative Dose-Related Reduction in Fracture Risk Achieved by Steroid-Dependent Asthmatic Adults after Substituting ICS for Past Prednisone Therapy

\begin{tabular}{|c|c|c|}
\hline \multirow[b]{2}{*}{ Cumulative Lifetime ICS Exposure } & \multicolumn{2}{|c|}{ Percent of Group with Normal Bone Density* } \\
\hline & Men $(n=26)$ & Women $(n=43)^{\dagger}$ \\
\hline$(10.1 \pm 5.5 \mathrm{yr})$ & Age $58.5 \pm 17.6(\mathrm{SD}) \mathrm{yr}$ & Age $60.8 \pm 9.6(\mathrm{SD}) \mathrm{yr}$ \\
\hline $\begin{array}{l}\leq 3 \mathrm{~g}^{\ddagger} \\
>3 \mathrm{~g}^{\ddagger}\end{array}$ & $\begin{array}{l}20 \\
40^{\S}\end{array}$ & $\begin{array}{l}50 \\
65^{\S}\end{array}$ \\
\hline
\end{tabular}

Adapted from Toogood $\mathrm{JH}$ et al. ${ }^{4}$

ICS $=$ inhaled corticosteroid.

*Lumbosacral bone mineral density Z-score (LBMD-Z): +4 to -1 .

${ }^{\dagger}$ All postmenopausal women received supplemental estrogen.

Median lifetime cumulative ICS exposure $=3 \mathrm{~g}$. Lifetime prednisone exposures were balanced equally between the higher and lower lifetime ICS exposure subgroups $(p=.87) .^{4}$

${ }^{\S}$ Larger lifetime exposures to ICS were associated with more normal LBMD-Z scores $(p=.002$ analysis of covariance) and a substantially larger number of patients with Z-scores indicating no increase in fracture risk. ${ }^{4}$

approved doses of a standard formulation of budesonide (without the adrenergic component) in four rather than two divided treatments each day can increase the antiasthmatic potency of the ICS as much as six to sevenfold (Figure 2$)^{19}$ and materially improve clinical outcomes ${ }^{20}$ without an accompanying increase in systemic risks or cost. ${ }^{19,20}$ For many patients, cost is likely to be a major determinant of whether ICS is prescribed or dispensed. ${ }^{21}$

For patients with mild persistent asthma, the combination of salmeterol plus fluticasone is more effective than the antileukotriene montelukast. ${ }^{17} \mathrm{On}$ the other hand, in patients with more severe asthma suboptimally controlled on low or moderate doses of ICS, high-dose zafirlukast may provide an effective nonsteroidal alternative to increasing the corticosteroid dose. ${ }^{22}$

For postmenopausal asthmatic women, an estrogen and progesterone supplement is recommended to reduce the risk of fracture. ${ }^{3}$ To maximize its bone-conserving effect, the estrogen replacement should commence soon after the advent of menopause.

Bisphosphonate therapy currently constitutes a first-line choice for the treatment or prevention of glucocorticoid-induced osteoporosis. ${ }^{3}$ Caution is advised with respect to its use in premenopausal women. ${ }^{23}$

\section{Applications of DXA in Clinical Practice}

Bone densitometry is deemed appropriate where the results of the test may reasonably be expected to directly influence patient management. ${ }^{1}$

Patients with at least one major or two minor risk factors for fracture are candidates for DXA (see Table 1). Systemic glucocorticoid therapy for more than 3 months is identified as a major risk factor. ${ }^{1,3}$ DXA is also recommended for asthmatic adults who require an ICS dose with a systemic potency equal to or exceeding that of $1.0 \mathrm{mg}$ BDP per day. ${ }^{9}$

However, regardless of the daily dose, DXA should be sought routinely in elderly asthmatic patients receiving ICS or prednisone treatment because a majority may exhibit a clinically important (and potentially treatable) increase in fracture risk. Furthermore, the degree of risk or nonrisk in a particular patient cannot be accurately estimated from any combination of the identifiable risk factors for osteoporosis and/or common clinical features of hypercortisonism and/or records of their current or past inhaled and/or oral corticosteroid exposure, with or without accompanying measurement of laboratory indices of bone metabolism. ${ }^{8}$

Contradictory findings have been reported as to the accumulative effects of prolonged ICS treatment on BMD. ${ }^{4-7,24,25}$ It has been suggested 

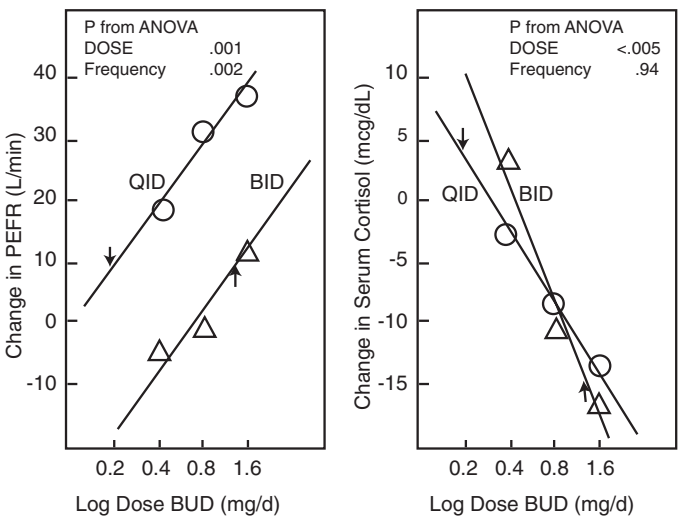

Figure 2 Comparison of the effects of two different dosing frequencies on the antiasthmatic (left panel) and systemic (right panel) responses to three graded per diem doses of budesonide (BUD) administered to 34 adults with moderate to severe unstable chronic asthma. ${ }^{19}$ The systemic effect of BUD was measured in terms of its impact on the 8:00 am serum cortisol level. Every patient took each of the six dose $\times$ dosing frequency combinations for the same length of time in a prospective, double-blind, balanced crossover protocol. To achieve an antiasthmatic response equivalent to that obtained with four BUD treatments per day, twice-daily treatment required a six- to sevenfold higher daily dose of BUD (arrows in left panel). This entailed a commensurate increase in the systemic toxicity of the inhaled corticosteroid (compare arrow symbols in the right panel). ANOVA = analysis of variance; PEFR = peak expiratory flow rate.

that patients with a cumulative lifetime exposure to ICS that exceeds $5.0 \mathrm{~g}$ should be candidates for DXA and, depending on the result, considered for preventive intervention to reduce their risk of fracture in later life. ${ }^{25}$ The preliminary measurement by DXA is important because some patients retain normal BMD values despite prolonged exposure to ICS therapy (see Figure 1 and Table $3)$. Therefore, they have no appreciable clinical need for active intervention with antiresorptive therapy.

A T-score value at or below -2.5 as determined by DXA indicates established osteoporosis and an important increase in fracture risk. ${ }^{2} \mathrm{~T}$ scores in the osteopenic range between -1 and -2.5 indicate a need for antiresorptive treatment to prevent further bone depletion. ${ }^{2}$

Where the diagnosis of osteopenia or osteoporosis has been confirmed by DXA and corrective bisphosphonate therapy initiated, the response to the bisphosphonate should be monitored by serial DXA examinations at intervals of about 1 to 3 years. ${ }^{3}$

It is not yet known whether the early introduction of long-term low-dose ICS therapy in young children with mild asthma may ultimately reduce the peak bone mass that they attain at maturity. A baseline DXA measurement may be appropriate in such patients when they attain 30 years of age - with appropriate follow-up depending on the result.

\section{Acknowledgments}

Special thanks to Maria Bettencourt for assistance in manuscript preparation and Peter B. Toogood, BSc (Eng), BSc (Pharm) for assistance in the literature review.

\section{References}

1. Khan AA, Brown JP, Kendler DL, et al. The 2002 Canadian bone densitometry recommendations: take home messsages. Can Med Assoc J 2002;167:1141-5.

2. Assessment of fracture risk and its application to screening for post-menopausal osteoporosis. Geneva: World Health Organization; 1994. WHO Technical Report Series No.: 843.

3. Brown JP, Josse RG, for the Scientific Advisory Council of the Osteoporosis Society of Canada. The 2002 clinical practice guidelines for the diagnosis and management of osteoporosis in Canada. Can Med Assoc J 2002;167 Suppl):S1-34.

4. Toogood JH, Baskerville JC, Markov AE, et al. Bone mineral density and the risk of fracture in patients receiving long-term inhaled steroid therapy for asthma. J Allergy Clin Immunol 1995;96:157-66.

5. Childhood Asthma Management Program Research Group. Long term effects of budesonide or nedocromil on children with asthma. N Engl J Med 2000;343:1054-63.

6. Lung Health Study Group. Effect of inhaled triamcinolone on the decline in pulmonary function in chronic obstructive pulmonary disease. N Engl J Med 2000;343:1902-9. 
7. Israel E, Bannerjee TR, Fitzmaurice GM, et al. Effects of inhaled glucocorticoids on bone density in pre-menopausal women. N Engl J Med 2001;345:941-7.

8. Toogood JH, Hodsman AB, Fraher LJ, et al. Serum osteocalcin and procollagen as markers for the risk of osteoporotic fracture in corticosteroid-treated asthmatic adults. J Allergy Clin Immunol 1999;104:769-74.

9. Boulet L-P, Becker A, Bérubé D, et al. Canadian asthma consensus report, 1999. Can Med Assoc J 1999;161 Suppl:S1-61.

10. Lau EMC, Woo J, Chan YH, Li M. Alendronate for the prevention of bone loss in patients on inhaled steroid therapy. Bone 2001;29:506-10.

11. Adachi JD, Bensen WG, Brown J, et al. Intermittent etidronate therapy to prevent corticosteroid-induced osteoporosis. N Engl J Med 1997;337:382-7.

12. Dawson-Hughes B. Bone loss accompanying medical therapies. N Engl J Med 2001;345:989-91.

13. Dubois EF, Roder E, Deakhuijzen E, et al. Dual energy x-ray absorptiometry outcomes in male COPD patients after treatment with different glucocorticoid regimens. Chest 2002;12:1456-63.

14. Dalsky GP, Stocke KS, Ehsani AA, et al. Weight bearing exercise training and lumbar bone mineral content in post-menopausal women. Ann Intern Med 1998;108:824-8.

15. Holt S, Suder A, Weatherall M, et al. Dose-response relation of inhaled fluticasone propionate in adolescents and adults with asthma: meta-analysis. BMJ 2001; 323:253-6.

16. Nelson HS, Busse WW, deBoisblanc BP, et al. Fluticasone propionate powder: oral corticosteroid-sparing effect and improved lung function and quality of life in patients with severe chronic asthma. J Allergy Clin Immunol 1999;103:267-75.

17. Nelson HS. Combination treatment with fluticasone/salmeterol in the treatment of asthma. J Allergy Clin Immunol 2001;107:397-416.

18. Pauwels RA, Lofdahl CG, Postma DS, et al. Effect of inhaled formoterol and budesonide on exacerbations of asthma. N Engl J Med 1997;337:1405-10.

19. Toogood JH, Baskerville JC, Jennings BJ, et al. Influence of dosing frequency and schedule on the response of chronic asthmatics to the aerosol steroid, budesonide. J Allergy Clin Immunol 1982;70:288-98.

20. Malo J-L, Cartier A, Merland N, et al. Four times a day dosing frequency is better than a twice-a-day regimen in subjects requiring a high dose inhaled steroid, budesonide, to control moderate to severe asthma. Am Rev Respir Dis 1989;140:625-8.

21. Kozyskj AL, Mustard CA, Cheang MS, et al. Income-based drug-benefit policy: impact on receipt of inhaled corticosteroid prescriptions by Manitoba children with asthma. Can Med Assoc J 2001;165:897-902.

22. Virchow JC Jr, Prasse A, Naya I, et al. Zafirlukast improves asthma control in patients receiving high dose inhaled corticosteroids. Am J Respir Crit Care Med 2000;162:578-85.

23. Recommendations for the prevention and treatment of glucocorticoid-induced osteoporosis, 2001 update. Arthritis Rheum 2001;44:1496-503.

24. Wong CA, Walsh LJ, Smith CJP, et al. Inhaled corticosteroid use and bone mineral density in patients with asthma. Lancet 2000;355:1399-403.

25. Sambrook PN. Inhaled corticosteroids, bone density, and the risk of fracture. Lancet 2000;355:1385. 